\title{
Dinâmica de Galáxia e Matéria Escura
}

\author{
Galaxy Dynamics and Dark Matter
}

\author{
Juliano Pereira Campos* \\ Departamento de Física - CCE - UFES \\ Campus Goiabeiras, 514 \\ Vitória - ES - 29075-910 \\ Rainer Madejsky ${ }^{\dagger}$ \\ Departamento de Física - UEFS \\ Campus Universitário, $\mathrm{Km}$ 03, BR 116 \\ Feira de Santana - BA - 44031-460
}

\begin{abstract}
Apresentamos neste trabalho uma análise da curva de rotação de galáxias espirais. Utilizamos alguns modelos analíticos na tentativa de obter uma expressão para a distribuição de massa da matéria escura, como uma possível solução para o "problema da matéria faltante". Uma combinação de modelos analíticos para descrever as distribuições de massa para matéria visível e matéria escura foram utilizados, respectivamente, a da Lei de Plummer e a da Esfera Isoterma. Salientamos que, mesmo tendo obtidos bons resultados, os modelos analíticos tratados neste trabalho não são os mais aperfeiçoados, e que nenhum outro fechou completamente o problema estudado.
\end{abstract}

\section{Palavras-chave: Dinâmica de Galáxias, Matéria Escura, Galáxias Espirais, Curva de Rotação, Astrofísica Extragaláctica.}

\begin{abstract}
We present in this article an analysis of curve of rotation for spiral galaxies. We use some analytical models to try get an expression for the distribution of dark matter mass as a possible solution to the "missing matter problem". A combination of two analytical models the Plummer law and Isotherm Sphere were used to describe the distribution of mass for visible and dark matter. We emphasize that the good results obtained here are independent of the analytical models considered to as anyone model had not completely solve the studied problem.

Keywords: Galaxy Dynamics, Dark Matter, Spiral Galaxies, Curve of Rotation, Extragalactic Astrophysics.
\end{abstract}

\section{INTRODUÇÃO}

Desde muito e muito anos atrás o ser humano além de ter ficado fascinado pelo espaço, procurou entendê-lo, e na atualidade não é diferente. Hoje, temos métodos e conhecimentos mais avançados que nos propiciam, cada vez mais, descobertas surpreendentes sobre o universo. E para viver melhor, cabe a nós tentar compreender a dinâmica da natureza e coope-

\footnotetext{
${ }^{*}$ Endereço Eletrônico: jpcampospt@hotmail.com

${ }^{\dagger}$ Endereço Eletrônico: madejsky@uefs.br
}

rar da melhor forma para a evolução do ser humano.

É da natureza humana, tentar compreender o que acontece ao seu redor. Para isso, o homem procura sistematizar e mensurar os acontecimentos e coisas que lhe são apresentadas, como por exemplo: massas de estrelas ou de galáxia, projetar saltos para alcançar satélites, dimensionar tamanho de constelações, enfim caracterizar o objeto apreciado. Uma dessas medidas é, justamente, objeto de estudo do nosso trabalho - a estimativa de massa de galáxias espirais.

A massa do universo por muito tempo teve 
uma característica eminentemente visível, ou seja, no cômputo da distribuição de massa dos objetos celestes se levava em conta apenas o que os olhos humanos e, com o passar do tempo, as lunetas puderam captar. Porém, essa tendência foi se modificando nos três últimos séculos, pois com o advento de telescópios ainda mais potentes e a aquisição de alguns métodos analíticos e computacionais, percebeu-se, entre outras coisas, que a teoria não estava compatível com a experimentação, vindo à tona a necessidade de repensar o problema do cálculo de massa dos corpos observados - chamado de "problema da matéria faltante".

A detecção de matéria escura em astronomia tem uma história relativamente longa. Começou em 1844 quando, por acaso, dois diferentes problemas de matéria escura foram identificados. Naquele ano notou-se que o planeta Urano tinha orbitado fora da posição calculada, por mais de 2 minutos de arco. No mesmo ano F.W. Bessel chamou a atenção para o movimento sinuoso da estrela Sirius.

O subseqüente problema de Urano conduziu, em 1845, J.C. Adams calcular com precisão a posição de um planeta hipotético cujo efeito gravitacional em Urano poderia ser responsável pelo seu movimento perturbado. E, em 1846, o astrônomo alemão Galle, observou a existência de um novo planeta (Netuno).

A primeira sugestão de uma possível distribuição generalizada de matéria escura gravitando em nossa galáxia foi feita por Kapteyn (1922) e Jeans (1922), seguidos por Lindbland (1926). Eles tentaram usar os movimentos observados de estrelas no plano da Galáxia para obter a densidade total de matéria próxima ao Sol. Kapteyn escreveu que "esta massa (matéria escura) não poderia ser excessiva".

O primeiro a afirmar que existia uma quantidade substancial de matéria escura próxima do Sol foi Oort $(1932,1965)$, que também utilizou os movimentos orbitais observados de estrelas. Ele obteve para a densidade total de matéria o valor de $\sim 0,2 M P c^{-3}$ (onde $M$ é a massa solar), que é quase duas vezes maior que a densidade de matéria observada próxima do Sol.

No início da década de 90 essas diferenças de quantidades de matéria escura presente próxima ao Sol (e no Universo) ainda era controvertida. Em 1991 Kuijken e Gilmore concluíram que "estas permanecem sem significativa evidência próxima ao Sol". Em contraste, Bahcall, Flynn e Gould (1992) sugeriram que um modelo sem matéria escura é inconsistente com os dados experimentais. Apesar da controvérsia, se faz necessária a determinação da quantidade de matéria escura devido a sua importância no estudo de modelos de dinâmica de galáxias, que irão desembocar na estrutura do Universo.

Frente ao que foi colocado no parágrafo anterior, objetivamos neste trabalho analisar alguns modelos analíticos que dão conta do cálculo de massa de objetos, como galáxias espirais, levando em consideração a matéria escura.

Com os modelos analíticos escolhidos: Plummer, Lei de Potência e da Esfera Isotérmica são estimadas as massas de galáxias espirais utilizando a gravitação Newtoniana.

\section{TEORIA GRAVITACIONAL}

A massa de uma galáxia espiral, aproximadamente, pode ser determinada pelo movimento de rotação das estrelas que estão em certa posição periférica da galáxia. Segundo a teoria, as velocidades de rotação das estrelas, a partir do ponto periférico, deveriam cair de acordo com a Lei de Kepler, porém as observações mostram que, por exemplo, a curva de rotação da nossa galáxia não cai rapidamente para raios maiores que os raios onde estão localizadas as estrelas periféricas. Uma das explicações para este fenômeno seria a existência de algum tipo de matéria que não conseguimos observar. Por esse motivo, se dá o nome a esta matéria de matéria escura; e ela explicaria a constância da curva de rotação através dessa curva se estima a massa de uma galáxia espiral, por exemplo.

No estudo da dinâmica de galáxias a distribuição de matéria, tanto escura quanto luminosa, é de fundamental importância para a de- 
terminação da estrutura de galáxia e da análise de colisão entre elas.

Supondo a interação entre galáxias como uma interação de corpos esféricos, e analisando essa interação através da Lei Gravitacional de Newton, para um corpo extenso de massas distribuídas continuamente, temos:

$$
\begin{aligned}
\vec{F}_{m} & =\sum_{i} \frac{m m_{i} G\left(\vec{r}_{i}-\vec{r}\right)}{\left|\vec{r}_{i}-\vec{r}\right|^{3}} \\
& =\iiint \frac{m G\left(\vec{r}_{i}-\overrightarrow{r^{\prime}}\right) \rho\left(r^{\prime}\right)}{\left|\vec{r}_{i}-\vec{r}^{\prime}\right|^{3}} d v^{\prime} .
\end{aligned}
$$

A energia potencial é dada por,

$$
V_{m}(\vec{r})=-\sum_{i} \frac{m m_{i} G\left(\vec{r}_{i}-\vec{r}\right)}{\left|\vec{r}_{i}-\vec{r}\right|}
$$

o potencial gravitacional é definido por

$$
\begin{aligned}
\psi(\vec{r}) & =\frac{V_{m}(\vec{r})}{m}=-\sum_{i} \frac{m_{i} G\left(\vec{r}_{i}-\vec{r}\right)}{\left|\vec{r}_{i}-\vec{r}\right|} \\
& =\iiint \frac{G\left(\vec{r}_{i}-\overrightarrow{r^{\prime}}\right) \rho\left(r^{\prime}\right)}{\left|\vec{r}_{i}-\vec{r}^{\prime}\right|} d v^{\prime}
\end{aligned}
$$

e

$$
\vec{g}(\vec{r})=\frac{\vec{F}_{m}}{m}=\iiint \frac{G\left(\vec{r}_{i}-\overrightarrow{r^{\prime}}\right) \rho\left(r^{\prime}\right)}{\left|\overrightarrow{r_{i}}-\overrightarrow{r^{\prime}}\right|^{3}} d v^{\prime},
$$

ou ainda,

$$
\vec{g}(\vec{r})=\nabla \psi(\vec{r})
$$

Se $\vec{g}=\nabla \psi$, então temos $\nabla(\nabla \psi)=\nabla^{2} \psi=$ $-4 \pi G \rho$, que é a equação de Poisson. Particularmente, $\operatorname{com} \rho=0$ implica que $\nabla^{2} \psi=0$, que é a equação de Laplace. (Symon 1982).

\section{MODELOS ANALÍTICOS}

\section{A. Modelos Analíticos para a Componente Visível}

Os modelos analíticos que serão usados neste trabalho são: o Plummer e o de Lei de Potência.

Com o auxílio da Lei de Gravitação acima citada, juntamente com os modelos analíticos, podemos obter caracterizações do potencial gravitacional que envolve galáxias. Iniciaremos nosso estudo buscando determinar a distribuição de massa e o potencial gravitacional dados por cada um dos modelos, depois encontraremos a curva de rotação para cada modelo, pois ela nos dará, indiretamente, a distribuição de matéria escura e visível numa galáxia.

A massa $M(R)$ será obtida da seguinte maneira:

$$
M(R)=4 \pi \int_{0}^{R} \rho\left(R^{\prime}\right){R^{\prime}}^{2} d R^{\prime},
$$

e pela Lei de Potência temos:

$$
\rho(R)=\rho_{0}\left(\frac{R_{c}}{R}\right)^{\alpha},
$$

onde $R_{c}$ é o raio central da componente visível, e $\rho_{0}$ é a densidade da galáxia no raio $R_{c}$.

$$
\psi=-\frac{4 \pi G \rho_{0} R_{c}^{\alpha}}{(3-\alpha)(2-\alpha)} R^{2-\alpha},
$$

onde (8) é potencial gravitacional que será exercido por um modelo de galáxia segundo o modelo da Lei de Potência.

A Figura 1 mostra duas curvas de densidade do corpo observado em função do raio, utilizando a Lei de Potência. A curva em vermelho (curva1) é dada por uma potência $\alpha=1$, enquanto que para a curva em azul (curva 2) $\alpha=4$. Vemos que para raios próximos do centro do corpo, a curva 1 se aproxima mais rápido de um valor finito, porém para raios com valores grandes a densidade cai mais lentamente. Enquanto que, na curva 2, a densidade do corpo para raios próximos do centro do corpo diverge, todavia a densidade cai rapidamente quando o raio do corpo aumenta; sendo assim, temos que atribuir valores convenientes para $\alpha$. 
Se fizermos $\alpha<3$ a massa, dada pela Eq. (6), no interior do raio $R$ será finita, porém a massa cresceria infinitamente com $R$; no entanto, quando $\alpha>2$ a diferença de potencial dada pela Eq. (7), entre $R$ finito e $R$ tendendo ao infinito, é finita. Assim, os melhores valores para $\alpha$ estão entre $2<\alpha<3$, satisfazendo as condições necessárias do nosso problema. Como a curva de rotação é dada por

$$
\nu_{r}=\sqrt{\frac{1}{3}} \sqrt{\frac{G M(R)}{R}},
$$

pela Eq. (9) temos que

$$
\nu_{r}=\sqrt{\frac{1}{3}} \sqrt{\left(\frac{4 \pi G \rho_{0} R_{c}^{\alpha}}{(3-\alpha)} R^{2-\alpha}\right)} .
$$

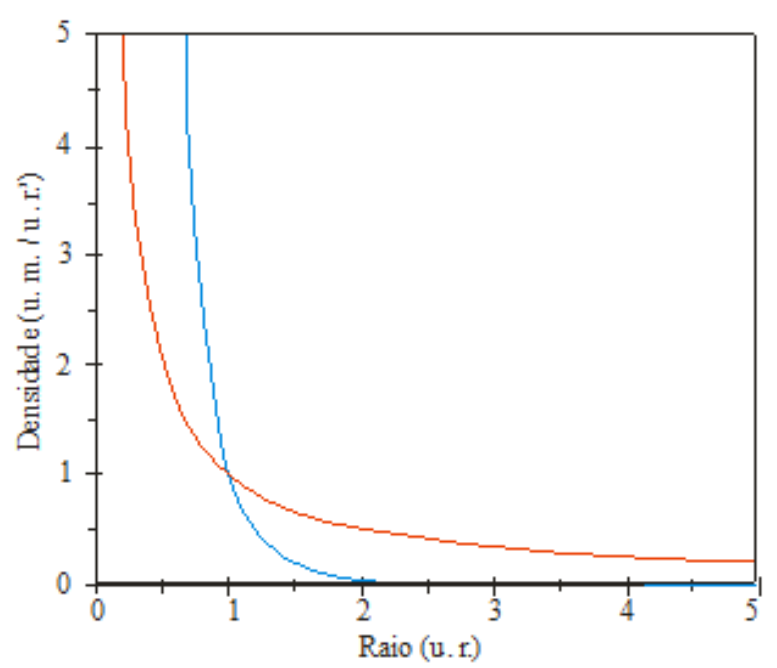

FIGURA 1: Curvas de densidade de massa de um corpo variando com o raio, tendo os valores de $\alpha$ iguais a 1 e 4 . Os valores utilizados mostram que se $\alpha$ for grande a densidade no centro do corpo é infinita e se for pequeno a desndidade do corpo para raios grandes vai aumentar muito.

Se fizermos $\alpha=1.9$ então $\nu_{r}=\sqrt{\frac{1}{3}} \sqrt{\left(\frac{4 \pi G \rho_{0} R_{c}^{1.9}}{1.1} R^{0.1}\right)} \Rightarrow \nu_{r} \propto \sqrt{R^{0.1}}$ e

$$
M(R)=\frac{4 \pi G \rho_{0} R_{c}^{1.9}}{1.1} R^{1.1} \Rightarrow M(R) \propto R^{1.1} .
$$

Para $\alpha=2.9, \nu_{r}$ será

$\nu_{r}=\sqrt{\frac{1}{3}} \sqrt{\left(\frac{4 \pi G \rho_{0} R_{c}^{2.9}}{0.1} R^{-0.9}\right)} \Rightarrow \nu_{r} \propto \sqrt{\frac{1}{R^{0.9}}}$,

e

$$
M(R)=\frac{4 \pi G \rho_{0} R_{c}^{2.9}}{0.1} R^{0.1} \Rightarrow M(R) \propto R^{0.1} .
$$

A Figura 2 mostra curvas que representam a dependência da densidade do corpo observando com o raio, dada pela Lei de Potência tendo $\alpha=(2.9,1.9$ e 2.5$)$, representadas em três cores diferentes: em vermelho temos $\alpha=1.9$, em verde $\alpha=2.9$ e $\alpha=2.5$ para a curva azul.

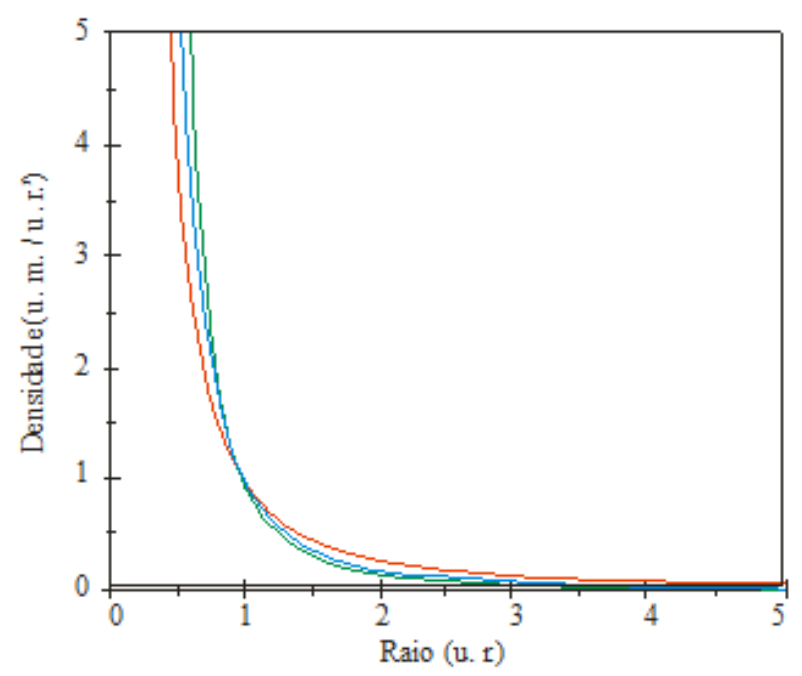

FIGURA 2: Curvas de densidade de massa de um corpo variando com o raio e tendo para $\alpha$ os valores $2.9,1.9$ e 2.5 .

A representação das curvas de rotação para os valores de $\alpha=(2.9,1.9$ e 2.5$)$ são mostradas na Figura 3. Das curvas plotadas, a que melhor representa a configuração de uma curva de rotação de uma galáxia espiral é a curva em vermelho. 


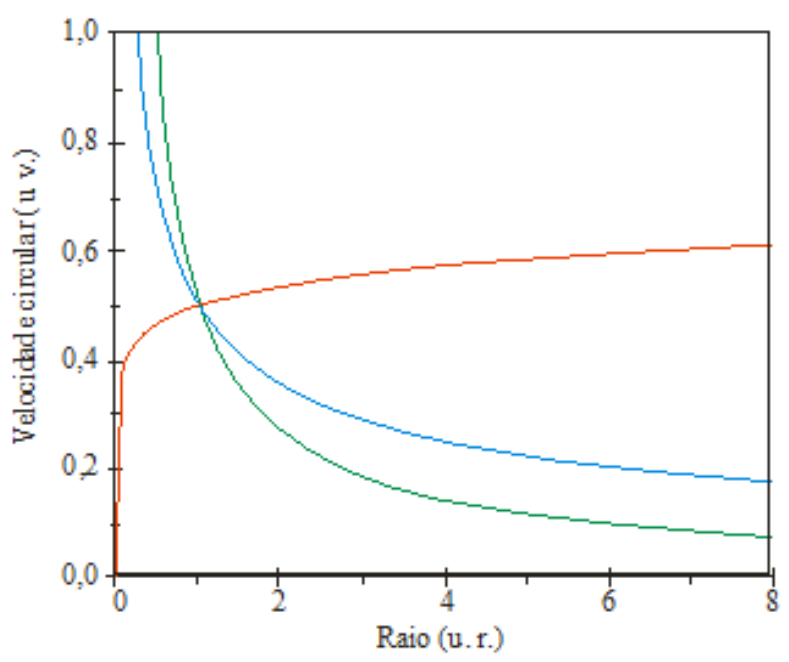

FIGURA 3: Curvas de rotação para valores de $\alpha=2.9,1.9$ e 2.5 dadas pela Lei de Potência, onde nota-se que das curvas traçadas apneas uma é compatível com a curva de rotação de uma galáxia espiral, aquela com $\alpha=1.9$.

Agora, para uma distribuição da densidade de matéria visível representada pela esfera de Plummer, temos:

$$
\rho_{\nu}(R)=\frac{3 M}{4 \pi} \frac{R_{c}}{\left(R^{2}+R_{c}^{2}\right)^{5 / 2}},
$$

onde $M$ é a massa total da matéria visível. A representação gráfica da distribuição de densidade dada pela esfera de Plummer está mostrada na Figura 4.

Da distribuição de densidade dada pela Eq. (15), representada na Figura 4, podemos notar que este modelo descreve de maneira mais realista a variação de densidade de um corpo com o raio, comparando com a distribuição de densidade dada pela Eq. (7) e representada na Figura 1, vemos que nesta, para um raio próximo do centro do corpo, o valor da densidade não converge para um valor finito, enquanto que, para a distribuição de Plummer, a densidade do corpo no centro é bem definida.

Calculando o potencial gravitacional, através da equação de Poisson devido à distribuição de densidade dada pela Eq. (15), temos:

$$
\psi_{\nu}(R)=-\frac{G M}{\left(R^{2}+R_{c}^{2}\right)^{1 / 2}} .
$$

Dessa forma obtemos o potencial de uma galáxia, onde sua distribuição de densidade é dada pelo modelo de Plummer. Podemos encontrar a distribuição de massa dada por esse modelo, e a partir dela determinar sua curva de rotação; da Eq. (6) temos:

$$
\begin{aligned}
M_{\nu}(R) & =4 \pi \int_{0}^{R} \frac{3 M}{4 \pi} \frac{R_{c}^{2}}{\left(R^{\prime 2}+R_{c}^{2}\right)^{1 / 2}} R^{\prime 2} d R^{\prime} \\
& =\frac{M R^{3}}{\left(R^{2}+R_{c}^{2}\right)^{3 / 2}} .
\end{aligned}
$$

Dessa forma, a curva de rotação dada por essa distribuição de massa será,

$$
\begin{aligned}
\nu_{r} & =\sqrt{\frac{1}{3}} \nu_{c}=\sqrt{\frac{1}{3}} \sqrt{\frac{G}{R} \frac{M R^{3}}{\left(R^{2}+R_{c}^{2}\right)^{3 / 2}}} \\
& =\sqrt{\frac{1}{3}} \sqrt{\frac{G M R^{2}}{\left(R^{2}+R_{c}^{2}\right)^{3 / 2}}} .
\end{aligned}
$$

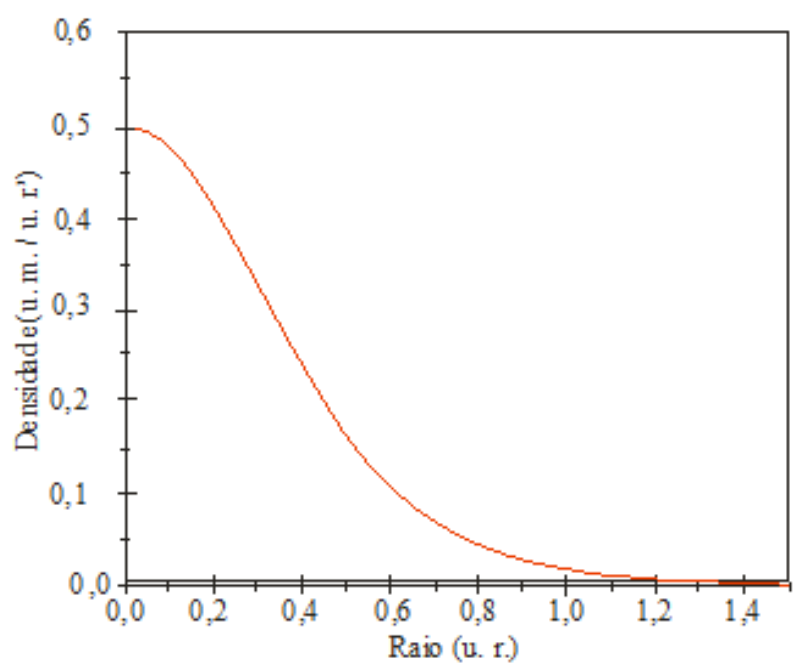

FIGURA 4: Curva de variação da distribuição da densidade de um corpo dada pelo modelo da esfera de Plummer.

\section{B. Modelo Analítico para a Componente Escura}

Para introduzirmos uma componente de matéria escura, trabalharemos com uma distribuição de densidade dada, aproximada- 
mente, por uma esfera isotérmica, ou seja,

$$
\rho_{e}(R)=\frac{\sigma^{2}}{2 \pi G\left(R^{2}+a^{2}\right)}, \quad R \leq R_{e},
$$

e

$$
\rho_{e}(R)=0, \quad R>R_{e},
$$

onde $\sigma$ é a velocidade de dispersão no Halo, e $a$ é o raio central da galáxia espiral. Calculando o potencial dado pela distribuição escura (ver Apêndice D) temos, para $R \leq R_{e}$

$$
\begin{aligned}
\psi_{e}(R)= & -\frac{2 \sigma^{2} a}{R}\left[\frac{R}{a}-\arctan \left(\frac{R}{a}\right)\right. \\
& \left.+\frac{R}{2} \ln \left(\frac{R_{e}^{2}+a^{2}}{R^{2}+a^{2}}\right)\right],
\end{aligned}
$$

para $R>R_{e}$

$$
\psi_{e}(R)=-\frac{2 \sigma^{2} a}{R_{e}}\left[\frac{R_{e}}{a}-\arctan \left(\frac{R_{e}}{a}\right)\right] .
$$

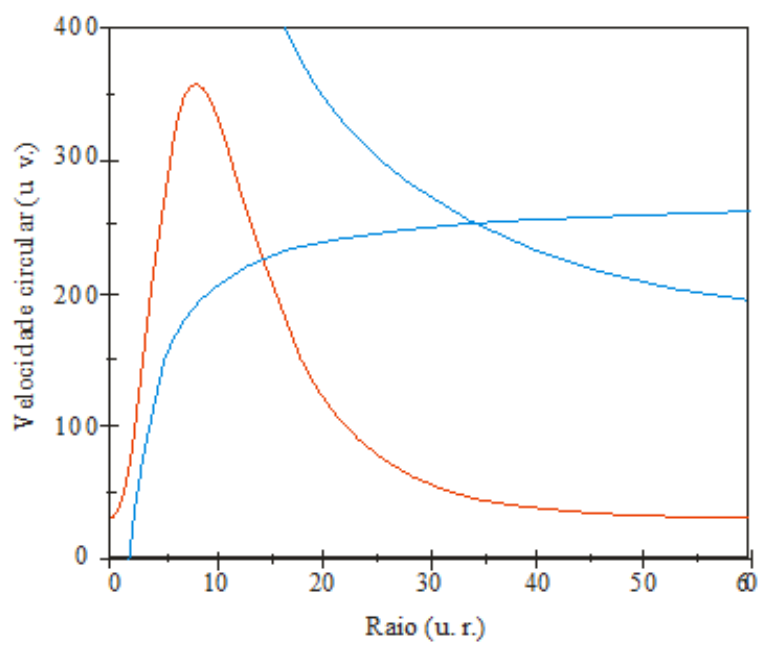

FIGURA 5: Curva da velocidade circular através do modelo de Plummer.

Para encontrarmos a curva de rotação da componente escura, faz-se necessário conhecer a distribuição de massa dada pela esfera isotérmica, então: para $R \leq R_{e}$

$$
\begin{aligned}
M_{e}(R) & =4 \pi \int_{0}^{R} \frac{\sigma^{2} R^{\prime 2}}{2 \pi G\left({R^{\prime}}^{2}+a^{2}\right)} d R^{\prime} \\
& =\frac{2 \sigma^{2} a}{G}\left[\frac{R}{a}-\arctan \left(\frac{R}{a}\right)\right],
\end{aligned}
$$

para $R>R_{e}$

$$
\begin{aligned}
M_{e}(R) & =4 \pi \int_{0}^{R} \frac{\sigma^{2} R^{\prime 2}}{2 \pi G\left(R^{\prime 2}+a^{2}\right)} d R^{\prime} \\
& =\frac{2 \sigma^{2} a}{G}\left[\frac{R_{e}}{a}-\arctan \left(\frac{R_{e}}{a}\right)\right] .
\end{aligned}
$$

Então, a curva de rotação será:

$$
\begin{aligned}
\nu_{e} & =\sqrt{\frac{1}{3}} \sqrt{\frac{G}{R} \frac{2 \sigma^{2} a}{G}\left[\frac{R}{a}-\arctan \left(\frac{R}{a}\right)\right]} \\
& =\sigma \sqrt{\frac{2}{3}} \sqrt{a\left[\frac{1}{a}-\frac{1}{R} \arctan \left(\frac{R}{a}\right)\right]} .
\end{aligned}
$$

\section{Representação Gráfica das Curvas de Rotação}

E a representação gráfica das curvas de rotação tanto para a componente visível, dada pela esfera de Plummer, como para a componente escura, dada pela esfera isotérmica, é mostrada na Figura 5.

A Figura 5 representa uma distribuição de massa para uma galáxia espiral onde se leva em conta tanto a parte visível como a matéria escura. As linhas em azul são linhas que representam a matéria escura, e elas devem ser vistas como uma única linha. Ou seja, partindo do eixo horizontal até o ponto de intersecção entre elas e deste seguindo adiante pela linha de baixo, pois a representação para a matéria escura se dar através de duas funções, uma até o raio escuro e a outra após o mesmo. A curva em vermelho representa a distribuição de massa para matéria visível.

A galáxia mostrada na Figura 6, representada por esta curva de rotação, tem $M=1.0$, 
$R_{c}=0.2, \sigma=2.1, \alpha=0.6$ e $R_{H}=4.0$. A massa escura é cerca de 6.2 unidades. A unidade de massa é $10^{11} \mathrm{M}$, unidade de comprimento é $10 \mathrm{kpc}$ e a unidade de tempo é 108 anos. Nestas unidades $G=4.497$, e as velocidades são dadas em unidades de $97.8 \mathrm{~km} / \mathrm{s}$.

Comparando os gráficos dados pelos modelos referidos no corpo do trabalho com o gráfico da Figura 6, evidenciamos a semelhança entre eles, apesar da simplicidade dos modelos utilizados aqui.

\section{CONCLUSÕES}

Os resultados encontrados utilizando os modelos de Plummer e da Esfera Isotérmica, apesar de não serem muito sofisticados, condizem com as observações e com resultados apresentados na literatura.

Apresentamos aqui umas das possibilidades de resolução do problema de matéria faltante utilizando a gravitação Newtoniana, todavia existem outras vertentes para a resolução do problema, que levam à modificação da lei gravitacional; por fim, a proposta do trabalho, de estimar a massa de galáxia levando em conta a matéria escura, é satisfeita.

\section{APÊNDICE A}

Para encontrarmos a intensidade do campo gravitacional devido a um corpo calculamos a seguinte integral,

$$
I=\iint \hat{n} \cdot \vec{g} d S
$$

como $\hat{n} \cdot \vec{g}=-\frac{1}{r^{2}} m G \cos \theta$ e $d \Omega=\frac{1}{r^{2}} \cos \theta d S$, onde $d \Omega$ é o elemento infinitesimal de ângulo sólido, então

$$
I=\iint \hat{n} \cdot \vec{g} d S=-\iint m G d \Omega=-4 \pi m G .
$$

Para uma superfície envolvendo um conjunto de massas $m_{i}$ teremos

$$
I=\iint \hat{n} \cdot \vec{g} d S=-\sum_{i} 4 \pi m_{i} G
$$

porém, pelo teorema da divergência de Gauss

$$
\begin{aligned}
I & =\iint \hat{n} \cdot \vec{g} d S=-\iiint 4 \pi G \rho d v \\
& =\iiint \nabla \cdot \vec{g} d v,
\end{aligned}
$$

$\log 0$

$$
\begin{aligned}
& \iiint(4 \pi G \rho+\nabla \cdot \vec{g}) d v=0 \\
& \Rightarrow \nabla \cdot \vec{g}=-4 \pi G \rho .
\end{aligned}
$$

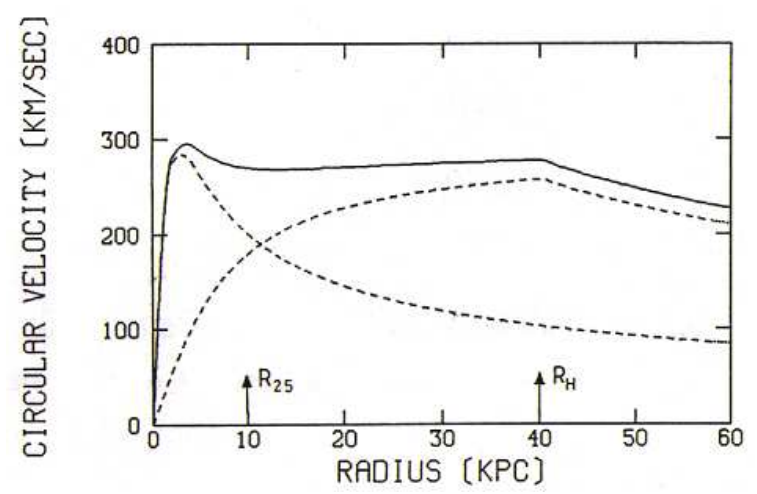

FIGURA 6: Curva de rotação de modelo de galáxia. O plano da velocidade rotacional é de $290 \mathrm{~m} / \mathrm{s}$, e a razão $M_{e s c} / M_{v i s}$ dentro de $R_{25}\left(=5 x R_{c}\right)$ é 0.8 . As linhas pontilhadas representam as curvas de rotação para as componentes visível e escura do modelo. O halo escuro é trucado em $R_{H}$. Para raios externos, a curva de rotação tem um declínio Kepleriano.

\section{APÊNDICE B}

Calculando a massa

$$
\begin{aligned}
M(R) & =4 \pi \int_{0}^{R} \rho_{0} R_{c}^{\alpha}{R^{\prime 2-\alpha}}^{2-} R^{\prime} \\
& =\frac{4 \pi \rho_{0} R_{c}^{\alpha}}{3-\alpha} R^{3-\alpha}
\end{aligned}
$$

o potencial gravitacional devido a esta distribuição será dado pela integração da equação 
de Poisson,

$$
\begin{aligned}
\nabla^{2} \psi & =\frac{1}{R^{2}} \frac{d}{d R}\left(R^{2} \frac{d \psi}{d R}\right)=-4 \pi G \rho \\
& =-4 \pi G \rho_{0}\left(\frac{R_{c}}{R}\right)^{\alpha}
\end{aligned}
$$

ou seja,

$$
\begin{aligned}
R^{2} \frac{d \psi}{d R} & =-4 \pi G \rho_{0} R_{c} \int_{0}^{R} R^{\prime 2-\alpha} d R^{\prime} \\
& =\frac{4 \pi G \rho_{0} R_{c}^{\alpha}}{3-\alpha} R^{3-\alpha}
\end{aligned}
$$

integrando-a uma vez mais obtemos

$$
\begin{aligned}
\psi & =-\frac{4 \pi G \rho_{0} R_{c}^{\alpha}}{3-\alpha} \int_{0}^{R} R^{1-\alpha} d R^{\prime} \\
& =-\frac{4 \pi G \rho_{0} R_{c}^{\alpha}}{(3-\alpha)(2-\alpha)} R^{2-\alpha} .
\end{aligned}
$$

\section{APÊNDICE C}

Calculamos o potencial gravitacional devido a distribuição de densidade a seguir através da equação de Poisson

$$
\begin{aligned}
\nabla^{2} \psi_{\nu}(R) & =\frac{1}{R^{2}} \frac{d}{d R}\left(R^{2} \frac{d \psi_{\nu}(R)}{d R}\right)=-4 \pi G \rho_{\nu} \\
& =-4 \pi G \frac{3 M}{4 \pi} \frac{R_{c}}{\left(R^{2}+R_{c}^{2}\right)^{5 / 2}} . \quad(\mathrm{C} 1)
\end{aligned}
$$

Assim, integrando-a uma vez

$$
\begin{aligned}
R^{2} \frac{d \psi_{\nu}(R)}{d R}= & -3 M G R_{c}^{2}\left[\frac{R}{3\left(R_{c}^{2}+R^{2}\right)^{3 / 2}}\right. \\
& \left.+\frac{1}{3 R_{c}^{2}} \frac{R}{\left(R_{c}^{2}+R^{2}\right)^{1 / 2}}\right], \quad(\mathrm{C})
\end{aligned}
$$

e por fim integrando-o uma vez mais conseguimos

$$
\psi_{\nu}(R)=-\frac{G M}{\left(R_{c}^{2}+R^{2}\right)^{1 / 2}} .
$$

Para o cálculo do potencial devido a uma distribuição de esfera isoterma partimos da expressão

$$
\begin{aligned}
\nabla^{2} \psi_{e}(R) & =\frac{1}{R^{2}} \frac{d}{d R}\left(R^{2} \frac{d \psi_{e}(R)}{d R}\right)=-4 \pi G \rho_{e} \\
& =-4 \pi G \frac{\sigma^{2}}{2 \pi G\left(R^{2}+a^{2}\right)}, \quad \text { (D1) }
\end{aligned}
$$

que rearrumada e integrada conduz a

$$
\begin{aligned}
R^{2} \frac{d \psi_{e}(R)}{d R}= & -2 \sigma^{2}\left[\int_{0}^{R} \frac{\left(R^{\prime 2}+a^{2}\right)}{\left(R^{\prime 2}+a^{2}\right)} d R^{\prime}\right. \\
& \left.-\int_{0}^{R} \frac{a^{2}}{\left(R^{\prime 2}+a^{2}\right)} d R^{\prime}\right],
\end{aligned}
$$

ou ainda,

$$
R^{2} \frac{d \psi_{e}(R)}{d R}=-2 \sigma^{2} a\left[\frac{R}{a}-\arctan \left(\frac{R}{a}\right)\right] .
$$

Realizando mais uma integração alcançamos o potencial devido a distribuição da esfera isoterma:

$$
\begin{aligned}
\psi_{e}(R)= & -2 \sigma^{2} a \int_{0}^{R}\left[\frac{1}{R^{\prime} a}\right. \\
& \left.-\frac{1}{{R^{\prime}}^{2}} \arctan \left(\frac{R^{\prime}}{a}\right)\right] d R^{\prime},
\end{aligned}
$$

ou seja, para $R \leq R_{e}$,

$$
\begin{aligned}
\psi_{e}(R)= & -\frac{2 \sigma^{2} a}{R}\left[\frac{R}{a}-\arctan \left(\frac{R}{a}\right)\right. \\
& \left.+\frac{R}{2} \ln \left(\frac{R_{e}^{2}+a^{2}}{R^{2}+a^{2}}\right)\right],
\end{aligned}
$$

enquanto que para $R>R_{e}$ temos

$$
\psi_{e}(R)=-\frac{2 \sigma^{2} a}{R_{e}}\left[\frac{R_{e}}{a}-\arctan \left(\frac{R_{e}}{a}\right)\right] .
$$

\section{APÊNDICE D}


[1] J. Binney, S. Tremaine, Galactic Dynamics. New Jersey: Princeton Series in Astrophysics (1987).

[2] D.M. Elmegreen, Galaxies and Galactic Structure. New Jersey: Prentice Hall (1998).

[3] S. Hawking, O Universo numa Casca de Noz. Tradução de Ivo Korytowski; Revisão Técnica Augusto Damineli. São Paulo: EDUSP (2002).

[4] W.J. Maciel, Astronomia e Astrofísica. São Paulo: IAGUSP (1993).

[5] K.S.F. Oliveira, M.F.O. Saraiva, Fundamentos de Astronomia e Astrofísica. Porto Alegre: UFRGS (1999).
[6] D.W. Sciama, Modern Cosmology and the Dark Matter Problem. New York: Cambridge Lecture Notes in Physics (1993).

[7] D.S.L. Soares, Investigations of Binary Galaxies. Groningen: Rijksuniversiteit (1989).

[8] K.R. Symon, Mecânica. Tradução Gilson Brand Batista. Rio de Janeiro: Editora Campus (1982).

[9] A.C.S. Friaça, E. Dal Pino, L. Sodré Jr., V. Jatenco-Pereira, Astronomia: Uma Visão Geral do Universo. São Paulo: EDUSP (2003).

[10] Página WEB: http://www.astro.if.ufrgs.br. 